\title{
Las interacciones de la niñez y adolescencia trabajadora en las calles de Xalapa, Veracruz, México: análisis de los mecanismos de control y estrategias de resistencia
}

The interactions of working children and adolescents in the streets of Xalapa, Veracruz, Mexico: analysis of control mechanisms and strategies of resistance

Arturo Narváez Aguilera

Sociología por la Universidad Veracruzana
Recibido el 29/01/2019

Revisión del 10/01 al 20/06/2019

Publicación 26/06/2019

\section{Resumen}

Para analizar las interacciones de las niñas, niños y adolescentes trabajadores en las calles de Xalapa, Veracruz, México, desde un enfoque sociológico, se abordan las categorías de mecanismos de control y la producción de estrategias de resistencia, como fundamentos conceptuales para explicar la tensión permanente entre la acción infantil trabajadora y la existencia de un régimen de control y regulación del trabajo infantil. Primero, se expone la operación de dispositivos y aparatos de control a diversas escalas, que transitan desde el umbral de una visión normativa y hegemónica en clave neoliberal del espacio público, hasta los tutelares-proteccionistas propios de la concepción moderna de la infancia. Segundo, se establece una conceptualización de las resistencias observadas en el sistema de interacción de la niñez y adolescencia trabajadora. Tercero, se describen los significados que tienen para los sujetos sociales la calle y su actividad económica. Finalmente, se explican las prácticas colectivas de resistencia, desde la noción de actores sociales por la sobrevivencia.

Palabras clave: infancia, niñez y adolescencia trabajadora, la calle, trabajo infantil, mecanismos de control, estrategias de resistencia. 


\begin{abstract}
To analyze the interactions of working children and adolescents in the streets of Xalapa, Veracruz, Mexico, from asociological perspective, the categories of the controlmechanisms and the production of resistance strategies are addressed, as conceptual foundations to explain the permanent tension between the child labor action and the existence of a regime of control and regulation of child labor. First, it exposes the operation of devices and control devices at various scales, which move from the threshold of a normative and hegemonic vision in a neoliberal key of the public space, to the protectionist-tutelares typical of the modern conception of childhood. Second, a conceptualization of the resistances observed in the interaction system of working children and adolescents is established. Third, the meanings that the street and its economic activity have for social subjects are described. Finally, the collective practices of resistance are explained, from the notion of social actors for survival.
\end{abstract}




\section{Introducción}

https://doi.org/10.35756/educaumch.v7i13.91

El texto que a continuación se presenta resume los principales hallazgos y resultados de la investigación denominada "Las interacciones de los niñosy adolescentes trabajadores en las calles: un análisis de los mecanismos de control, estrategias de resistencia y empoderamiento en la Zona Metropolitana de Xalapa, 1990-2014.", realizada en la Universidad Veracruzana.

El artículo está centrado en abordar la configuración de las interacciones de la niñez y adolescencia trabajadora en las calles con diversos actores y sus pares en Xalapa, Veracruz, México. El propósito fue analizar la producción de estrategias de resistencia de los sujetos sociales frente a la generación de mecanismos de control en el espacio público de las calles.

El objetivo central es exponer las experiencias y voces de niñas, niños y adolescentes trabajadores en las calles. Para ello, analizamos la multiplicidad de relaciones sociales que produce la acción infantil trabajadora en coexistencia con un régimen de controles y regulaciones institucionales y privados hacia el trabajo infantil. Postulamos que el fundamento ideológico de la regulación social hacia estas poblaciones, está centrado en la visión hegemónica de la infancia moderna
(Ariés, 1987), el principio de socialización en clave funcionalista (Parsons,1959) y el paradigma proteccionista-universalista del pensamiento liberal de los derechos humanos.

Cabe destacar que la inquietud de aproximarse al tema surge por el interés de poner en el centro del análisis la perspectiva de los actores sociales frente a su realidad social, ante la insatisfacción de explicaciones abolicionistas centradas en las causas y consecuencias que la actividad económica produce.

Con esta posición epistemológica, la orientación de la investigación implicó un recorrido exploratorio y de acercamiento a diversas perspectivas teóricas, conceptuales y metodológicas. En ello, los planteamientos de la sociología de la infancia ofrecieron pistas fundamentales para el trabajo la noción del concepto de infancia como categoría de análisis sociológico permite develar su condición social y construcción histórica, diferenciada por relaciones de poder (Gaitán, 2006).

En la misma línea, se retoma el enfoque orientado en el sujeto (Liebel, 2003), que permitió ampliar las miradas respecto a las representaciones sociales ${ }^{1}$ que tienen 
niñas, niños y adolescentes trabajadores sobre su posición diferenciada dentro de la estructura familiar y social, el significado de la calle, la re-significación de sus derechos en sus prácticas cotidianas y su noción de actores sociales en el espacio público.

Además, el concepto de interacciones fue una herramienta analítica relevante, que permitió tender un puente entre la acción social delaniñezyadolescencia trabajadora con las condiciones de la estructura social, económica e histórica que la regulan. Siguiendo con el argumento, la teoría de relaciones de poder desde la multiplicidad de relaciones de fuerza (Foucault, 2008), permitió operacionalizar el fundamento teórico para la interpretación de la evidencia empírica de la vida social en las calles.
En síntesis, el lector encontrará en el artículo el marco de las interacciones de la niñez y adolescencia trabajadora en Xalapa, Veracruz, a partir del análisis de las calles como espacios de cooperación y conflicto, donde se configuran los mecanismos de control y estrategias de resistencia.

1 Para Denise Jodelet (2008), las representaciones sociales son una forma de conocimiento específico, el saber de sentido común, cuyos contenidos manifiestan la operación de procesos generativos y funcionales socialmente caracterizados. En sentido más amplio, designa una forma de pensamiento social. Las representaciones sociales constituyen modalidades de pensamiento práctico orientados hacia la comunicación, la comprensión y el dominio del entorno social, material e ideal. En tanto que tales, presentan características específicas a nivel de organización de los contenidos, las operaciones mentales y la lógica" (p.474) 


\section{Los mecanismos de control en las calles de la ciudad de Xalapa}

Para establecer un marco espacial a la configuración de las interacciones de la niñez y adolescencia trabajadora en las calles, se optó por una estrategia metodológica basada en la construcción de información sobre la composición de las zonas y cruceros con poblaciones trabajadoras en las calles, que fue instrumentada en dos fases: la primera exploratoria y de observación no participante y la segunda de observación participante en los cruceros y zonas de trabajo ${ }^{2}$.

Las calles son espacios sociales heterogéneos, donde se disputan diversos capitales en "juego" en la relación de fuerzas (Bourdieu, 1990). En este caso, asociados a la sobrevivencia por parte de los grupos de trabajadores en calles y a las formas modernas y capitalistas de regulación social del espacio público y el trabajo informal.

En este contexto, la niñez y adolescencia trabajadora en las calles originan estrategias de resistencia que se traducen en una serie de prácticas individuales o colectivas de sobrevivencia, producidas como formas disidentes a los mecanismos de control institucional o de sus "pares", sustentadas en el conocimiento y habilidades de los sujetos sociales de la vida en calle.

Desde la escala micro-social de la

${ }^{2}$ Cabe destacar que se contó con el apoyo de jóvenes que han tenido más de diez años trabajando en las calles, los cuales narraron sus experiencias y saberes sobre la composición de cuatro zonas de referencia de niñas, niños y adolescentes trabajadores en las calles: Lázaro Cárdenas, Centro, Circuito Presidentes, Ruíz Cortines y 20 de Noviembre, con un total de 38 "cruceros" o esquinas de trabajo; también se realizó con educadores de calle de la organización Movimiento de Apoyo a Niños Trabajadores y de la Calle, (Matraca, A.C.). Para mayor información en el tema, acudir a la investigación en referencia. 
interacción cotidiana entre los grupos de trabajadores en las calles, la disputa está asociada al control de la "territorialidad" y uso de los cruceros como medio para la sobrevivencia. Cada espacio significa la posibilidad de acceder a ingresos económicos diferenciados, la construcción de la red de sobrevivencia, el reconocimiento de sus "pares" y la lucha de la legitimidad por la mayor condición de "vulnerabilidad" ante los consumidores de servicios callejeros.

Derivado de estas disputas, se configuran una serie de mecanismos de control que son prácticas y discursos adquiridos en la experiencia, los conocimientos y modos de vida en el trabajo en las calles, orientados a la apropiación de capitales para la sobrevivencia.

En el acontecer cotidiano, estos mecanismos son operados por liderazgos individuales y colectivos de cada crucero; las modalidades se expresan en la aceptación o exclusión del crucero o grupo, la asignación de la actividad económica (limpiar-parabrisas representa mayores ingresos que vender dulces), la calle o crucero permitido (cada espacio de trabajo de acuerdo a su ubicación y tránsito de vehículos o personas, produce ingresos diferenciados), la "profesionalización" de la actividad económica y la posición social frente a los diversos actores de la zona de trabajo.

Los grupos de trabajadores en calle son sujetos que producen relaciones sociales y de intercambio económico en el marco de la exclusión, marginación y discriminación. Para ello, establecen estrategias de venta y comercialización (precios, productos y lugares), configuran alianzas con otros actores comerciales y buscan posicionarse como referentes para sus consumidores ocasionales a partir de la apropiación simbólica del espacio público.

Sin embargo, en el espacio público no solo operan los mecanismos de control asociados a la sobrevivencia. En otra escala, se identifican también los dispositivos estatales de regulación tutelar-proteccionista que apuntan a la disciplina de la vida infantil trabajadora.

La triada infancia-trabajo-calle, representa una afrenta a las concepciones morales de la sociedad contemporánea y al discurso de protección a los derechos humanos; además, de la condición del modelo bipolar dominante de la infancia como "víctimas o delincuentes" (Parazelli, 2002). 
Respecto a los dispositivos del uso y función del espacio público, operan de diversos aparatos gubernamentales, fundamentalmente policías e inspectores. La condición de personas trabajando en las calles en la informalidad, es concebida como una situación de "riesgo" a la normalización de la vida social y del bien público, este último, construido en la noción de la ciudad como centro para el intercambio del mercado capitalista que se asocia a la modernidad, la imagen urbana, la formalidad y regulación de la actividad económica.

Por ello, las poblaciones trabajadoras en las calles representan el blanco de intervención de la gubernamentalidad moderna (Foucault,2006). Desde la noción de grupos sociales "irregulares" y "peligrosos", el tratamiento se produce por medio de mecanismos punitivos o de institucionalización que se traducen en acciones de retiro forzado ${ }^{3}$, hostigamiento y detenciones arbitrarias: una forma de intolerancia selectiva (Wacquant,2006).

De manera paralela, el régimen tutelar-proteccionista, operado por los aparatos gubernamentales de asistencia social. Instrumentan una serie de políticas y acciones, bajo el argumento de las condiciones de "desprotección" y "vulnerabilidad" de la niñez y adolescencia trabajadora en las calles. El principio ideológico se basa en la visión universalista/occidental de los derechos de la niñez, el principio de socialización, la concepción moderna de la infancia, el adultocentrismo como forma de relación asimétrica entre adultos-niñez y la traducción local del enfoque de erradicación del trabajo infantil impulsado por organismos internacionales.

En el centro de este régimen, se encuentra

${ }^{3}$ La Comisión de Derechos Humanos del Distrito Federal en la Recomendación 23/2009, define limpieza social como "el retiro de las personas non gratas de ciertos lugares, sin que medie justificación legal alguna [...] la razón para retirar [les es] sólo por su condición de calle". 
la vigilancia y sanción de la crianza de las familias en condiciones de marginación social y económica, concebidas como obstáculo para el bienestar y desarrollo infantil hacia la vida adulta (Pilotti, 2001). En ello, la ampliación de la función tutelar del Estado se objetiva en un conjunto de mecanismos asistenciales, administrativos y legales -bajo el modelo de la doctrina de la situación irregular-, tendientes a la criminalización y separación de las familias, la institucionalización como mecanismo disciplinario de la vida de la niñez y adolescencia trabajadora.

Las acciones se materializan en una serie de programas de asistencia y protección a los derechos de la niñez y adolescencia trabajadora que, de manera cíclica, se enfocan en la erradicación del trabajo infantil, influenciado por tratados internacionales y convenios que el Estado mexicano asume en la cooperación y asistencia técnica con organismos internacionales como la Organización Internacional del Trabajo (OIT). Dicho enfoque, configura una forma de intervención moral (Hard y Negri, 2000) en la producción de bases normativas y jurídicas de orden nacional y estatal, tendiente al control de los significados sobre el "deber ser" de la niñez y adolescencia trabajadora.

Las denominadas "circunstancias especialmentedifíciles"sonelfundamento de un proceso secuencial de acciones públicas (detección, retiro, asistencia, canalización, institucionalización y devolución), instrumentadas por diversos actores gubernamentales, con el argumento de la protección infantil. Dicho mecanismo tutelar-proteccionista, actúa bajo el principio de "medidas de salvamento" de la desprotección de la niñez y adolescencia que trabaja en las calles, incluyendo el uso de la fuerza pública en la etapa de detección y retiro del sector. 


\section{Categorías de estrategias de resistencia de la niñez y adolescencia trabajadora}

Hasta ahora, se han analizado los diversos mecanismos de control que operan en el espacio público de las calles a diversas escalas. Es relevante mencionar que los hallazgos de la investigación apuntan sus coordenadas de análisis y evidencia empírica en el reconocimiento de niñas, niños y adolescentes trabajadores en las calles como actores sociales, que generan prácticas colectivas de resistencia, resultado de las trayectorias y conocimientos acumulados del trabajo en calle.

Existe una heterogeneidad de estrategias que la niñez y adolescencia trabajadora utilizan para sortear los diversos controles institucionales, sociales, grupales e incluso familiares, las cuales fueron conceptualizadas por categorías en los hallazgos de la investigación y se expresan de la siguiente manera:

a) Estrategias de confrontación: prácticas de resistencia directa, que responden a los mecanismos de control por violencia institucional, social o familiar.

b) Estrategias de mediación, colaboración y negociación: prácticas de resistencia, que identifican la posición ante los mecanismos de control, y como respuesta, son formuladas desde la noción del establecimiento de acuerdos y compromisos que, de forma negociada, producirán beneficios e intereses tangibles en el corto, mediano y largo plazo.

c) Estrategias de participación

C1- Simulada: prácticas de resistencia, bajo el principio de la racionalidad instrumental de aceptar participar en procesos o temas del interés de los otros. C2- Protagónica: prácticas de resistencia, donde se asume una posición de actores o sujetos de derechos, que determinan la toma de decisiones y los temas de su interés.

d) Estrategias de sumisión-vulnerabilidad: prácticas de resistencia, donde la vulnerabilidad y desprotección se asumen de manera racional, como un medio para obtener beneficios inmediatos.

Es importante resaltar, que la producción de estas estrategias no es excluyente; por 
el contrario, las estrategias se encuentran articuladas y continuas de acuerdo a cada interacción específica de la niñez y adolescencia trabajadora con los diversos actores. Es relevante exponer que, las mismas, se configuran en diversos componentes de la acción infantil, como son los siguientes: el significado que tiene para el grupo social el trabajo infantil, la movilidad y adaptación en sus trayectorias por el espacio público, la comunicación colectiva, los espacios disidentes y la organización colectiva.

\section{El trabajo infantil desde la perspectiva de la niñez y adolescencia trabajadora en Xalapa}

Algunos de los hallazgos más significativos sobre las representaciones sociales que tienen las niñas, niños y adolescentes trabajadores sobre el trabajo infantil, difieren de las construcciones adultocéntricas enmarcadas por el discurso moralizante sobre el tema. Por el contrario, sus saberes acumulados en sus trayectorias individuales y colectivas en las calles, les permite discernir entre los beneficios y los riesgos que les plantea el trabajar en las calles. Por ello, es posible afirmar que la calle y su actividad económica tienen un significado ambivalente para el grupo social: libertad y hostilidad.

Inicialmente, el significado de trabajar en las calles no se concibe como una separación entre la actividad económica informal y su vida social; por el contrario, es interiorizado como una tarea cotidiana, que les permite la posibilidad de libertadrecreación en el espacio público $y$, fundamentalmente, la opción de poder acceder a recursos económicos materiales y simbólicos para apoyar a sus familias y acceder a satisfactores inmediatos.

Existe un matiz importante a destacar, respecto a la identidad colectiva como trabajadores en la calle. En el caso de la niñez trabajadora, los relatos expresan que no existe una identidad como trabajadores; porque, el trabajo es concebido como una actividad formal que se desarrolla en espacios comerciales cerrados: "los niños trabajadores son los que trabajan en las tiendas o en el $\mathrm{Chedra}^{4}$, nosotros jugamos en las calles, vendemos rosas y nos dan dinero" (Niña 1, 8 años, vende rosas).También, destaca en la observación, que esta disociación responde a una estrategia de resistencia ante las amenazas de retiro por parte de autoridades locales; en ello, el autoreconocimiento como niños trabajadores 
implicaría riesgos asociados a la separación de su entorno familiareinstitucionalización. En el caso de los adolescentes trabajadores, el trabajo significa la posibilidad de mantener sus estudios y apoyar a sus familias; percepción que responde al cambio en su estatus familiar por su aporte económico y al proceso de diferenciación con los "otros", los no productivos: "trabajamos en las calles para ganar dinero; de que estén robando mejor se dedican ahí, para que no anden en la calle; porque hay gente que anda en la calle, no andan haciendo nada y se drogan, mejor se deberían de poner así como nosotros a "chambear"'"'!' (Adolescente varón 1, 15 años, limpiaparabrisas).

En la misma línea de análisis, se encuentra la relación diferenciada que establecen entre las actividades que realizan en el ámbito doméstico y las ejercidas en el espacio público. Las primeras son concebidas como elementos de mayor desgaste y apatía, fundamentalmente, por la diversidad e intensidad de las mismas, que van desde la limpieza doméstica, lavado de su ropa y en la mayoría de los casos, asistir en la compra de productos en la tienda o con los vecinos.

Por el contrario, vender en las calles les permite tomar decisiones para realizar otras actividades libremente. "En el parque tengo más libertad de juego, me columpio en la viborita ${ }^{6}$ que está allí, me subo a los juegos" (Niña 2, 10 años, vende rosas-dulces); mientras que las actividades domésticas son impuestas por los adultos y existe una permanente vigilancia de la autoridad familiar dentro de espacios de vivienda limitados. La denominada "ayuda" de los niños y adolescentes en la vida doméstica no es vista como una actividad que produzca

4 El término alude a un supermercado en la ciudad de Xalapa, donde han trabajado adolescentes como empaquetadores de los víveres.

5 Para los trabajadores en las calles, "chambear" significa la acción de trabajar.

${ }^{6}$ La "viborita" es un juego en un parque público. 
beneficios inmediatos "en mi casa todos me ponen a hacer cosas-mi mamá y mis hermanos grandes-y no me compran nada, mejor yo me compro dulces con las rosas que vendo" (Niña 3, 8 años, vende rosas y dulces). En este sentido, la venta en las calles se percibe como una alternativa para ganar "dinero", que se traduce en satisfactores y además la aleja de manera temporal de la violencia familiar y la precariedad doméstica.

Otro de los elementos trascendentes para comprender los significados de la niñez y adolescencia trabajadora, es el ingreso que les produce su actividad económica en las calles, asociado directamente con la posición que les brinda en la estructura familiar, social e incluso escolar. Al respecto, el trinomio ganar, tener y manejar dinero (Domic, 2000), tiene como punto de partida la apropiación de las estrategias de acceso a ingresos diferenciados, resultado de un proceso paulatino de aprendizajes y prácticas colectivas sobre los lugares, las formas, los riesgos y el análisis de los consumidores potenciales.

La capacidad colectiva para acceder a recursos económicos y capitales simbólicos- en el marco de marginación y pobreza-,se traduce en el autoreconocimiento de sus conocimientos, esfuerzos físicos, conocimiento del entorno y las interacciones con diversos actores como estrategias de sobrevivencia. Estas últimas asociadas con sus habilidades de trabajar en contextos especialmente difíciles marcados por la discriminación, la competencia en los cruceros o parques, los mecanismos de control tutelar-proteccionista, los riesgos de las calles (físicos, violencia social, climatológica), la vigilancia permanente de policías e inspectores, entre otros. Todo ello imprime el valor que las niñas y adolescentes le dan al ingreso, producto de su esfuerzo y exposición a los avatares de la calle.

En los componentes de tener y manejar dinero, las implicaciones están asociadas a la posición que adquieren en el espacio escolar y familiar. En el ámbito doméstico, el aporte a la economía familiar tiene un sentido y significado simbólico para la niñez y adolescencia trabajadora; les otorga una posición y legitimidad en la organización familiar, les brinda un sentimiento de seguridad respecto a la posibilidad de adquirir y satisfacer necesidades de la vida familiar $y$, en algunos casos, les permite debatir con los 
adultos sobre la dirección de los ingresos propios, decidir sobre la adquisición de servicios privados (internet, cable), compras de aparatos electrónicos del hogar y responder a contingencias familiares (enfermedades, deudas y accidentes).

Respecto a la relación con el ámbito escolar, tener y manejar dinero coloca a la niñez y adolescencia trabajadora en las calles en una posición ambivalente con respecto a sus pares $u$ otros grupos en las calles, a diferencia de los niños $y$ adolescentes que no trabajan, que dependen del aporte económico de sus padres y con quienes interactúan en el espacio de la escuela. El grupo de trabajadores en la calle establece una resignificación del estatus escolar basado en el manejo de ingresos; esta posición de poder-como respuesta a la discriminación, exclusión social y educativa-, les permite demostrar objetivamente su autonomía y capacidad económica frente a los "otros", a partir de acceder a la compra de mayores productos en los espacios de "recreo" o a la salida de clases e incluso llegan a "invitar" dulces y comidas a sus compañeros, como lo afirma una adolescente:

"yo en mi bolsa siempre ando trayendo dinero, siempre que vamos a la escuela y siempre traemos dinero, nunca andamos sin ni un peso, lo que queremos comer, lo que nosotros queremos siempre lo tenemos, esa es nuestra ventaja en la calle-que si queremos un hot dog lo tenemossiempre lo tenemos todo, nosotros tenemos más dinero que los niños de la escuela, luego, hasta se sacan de onda- de dónde sacan tanto dinero-tenemos nuestra morralla ${ }^{7}$, nosotros somos los que mandábamos en la escuela-se podría decir- luego les digo que sí no quieres una torta y se las invito, como tenemos hasta 500 libres." (Adolescente mujer 2, 12 años, vende rosas).

En síntesis, trabajar en las calles, significa la posibilidad de libertad-empoderamiento en su toma de decisiones; la calle se convierte, en una posibilidad emancipadora de la vida infantil frente al mundo adulto; significa su actividad económica en el auto-reconocimiento de sus conocimientos, habilidades y estrategias que les brinda trabajar en la calle. Sin embargo, de manera permanente, es el contexto de violencia 
social y la vigilancia-regulación de su actividad con lo que deben de sortear; de ahí que, la actividad económica, les exige mayores niveles de organización y resistencia colectiva.

En el significado de hostilidad, conciben que su trabajo les produce riesgos, desgaste físico y frustración ante los bajos ingresos. Sin embargo, el factor que les produce mayor tensión colectiva es tener que desafiar los mecanismos de control por violencia social y tutelarproteccionistas de regulación del trabajo infantil; no solo por las implicaciones de obstaculizar su acceso a ingresos y satisfactores inmediatos, sino porque los primeros ponen en riesgo su vida o integridad física y los segundos atentan contra los sentimientos de la relación maternal y paternal, al instrumentalizar una serie de medidas jurídicas y asistenciales, tendientes a la separación familiar y la detención de las figuras emocionales de la niñez y adolescencia.

${ }^{7}$ El término alude a las monedas que traen consigo la niñez y adolescencia trabajadora, producto de su trabajo en las calles. 


\section{Resistencias de la niñez y adolescencia trabajadora en la interacción en las calles}

Analizar los mecanismos de control que operan en las calles a diversas escalas, implica develar la coexistencia de la producción de estrategias de resistencia en las condiciones determinadas (Foucault,1994); las cuales, en las interacciones de la niñez y adolescencia trabajadora en Xalapa, tienen como marco de referencia conceptual los significados que tiene el espacio público y el trabajo infantil para el grupo social.

Un primer abordaje se explica desde el componente de movilidad y trayectorias en el espacio público. Se afirma que las transiciones en las calles no son fortuitas, responden a las experiencias y conocimiento profundo de la composición de cada crucero o zona de trabajo, la diversidad de actores y sortear los riesgos y controles en cada dimensión social del espacio.

Ante los mecanismos de hostilidad, que van desde el carácter exponencial de peligro por violencia social, pasando por el umbral de que sus productos sean incautados por inspectores, hasta ser retirados o llevados a casas de asistencia social por los tutelares-proteccionistas, la niñez y adolescencia trabajadora se organiza por grupos sociales y familiares para vender sus productos. Reconocen la importancia de transitar los espacios de venta acompañados por al menos dos integrantes; de la misma forma, se transportan en grupo de sus casas a los lugares de venta y viceversa; previamente, han identificado espacios de protección o disidentes, que funcionan para encontrarse en caso de haberse dispersado (correr) para esconderse del hostigamiento.

Como estrategias de comunicación, utilizan los"silbidos" para formular un aviso de la proximidad de un riesgo; hablar con el lenguaje de la calle como símbolo de identidad colectiva; compartir de manera constante las situaciones del día a día en las calles y cuando termina uno primero de vender, los otros le ayudan a terminar, como gestos de solidaridad colectiva "con mis primas, con amigos que están vendiendo, con mi hermana: jugamos, vendemos y nos ayudamos" (Niña 2, 10 años, vende rosas y dulces). 
También las trayectorias en el espacio público cumplen la función de construir su red de apoyo con actores diferenciados privados y sociales; cada uno responde a necesidades e intereses específicos, para acceder a recursos diversos: protección, salud, educación, alimentación, vestido, juegos, útiles, zapatos. Al respecto, una adolescente menciona:

"Muchas puertas abiertas, la calle es generosa, te regalan ropa, zapatos, comida y dinero... yo quiero unos zapatos y me pongo afuera de la Zapatería X- el dueño me conoce- y me regala pa mí y mis hermanos, sí quiero ropa nueva, bajo a la tienda que me conocen, si quiero consultas voy a Matraca, en Caritas del centro comía, cuando quiero mis útiles, me pongo en la calle con mi lista y hay gente buena y me los compra." (Adolescente mujer 3, 15 años, vende tamales).

Otro de los componentes centrales, es la estrategia de "vulnerabilidad" asumida de manera racional y adaptativa a las condiciones del contexto social, como alternativa frente a la discriminación $y$ medio para acceder a recursos $y$ beneficios inmediatos con diversos actores. Destacan los consumidores de servicios callejeros, con los cuales los actores infantiles producen diversas estrategias de persuasión y negociación, como práctica colectiva que potencializa el espectro de acceso a ingresos. Además de incorporar otras estrategias, como el discurso emotivo, elaborar poemas (los que venden rosas) o solicitar comida. En el caso de los limpiaparabrisas, las estrategias hacia el sector de automovilistas son el discurso amable, no subirse a los autos o realizar el servicio con la promesa de un pago posterior.

Finalmente, en las micro-interacciones con poblaciones callejeras ${ }^{8}$ y trabajadoras, la mayoría de las niñas, niños y adolescentes trabajadores, buscan sortear a los grupos de jóvenes y adultos que trabajan o viven en las calles “[...] Con personas más grandes no nos llevamos, porque ellos no nos hablan, nosotros tampoco, nos dicen muchas groserías." (Niña 4, 9 años, vende dulces). Para ello, desarrollan sus actividades económicas principalmente en la venta de productos, en zonas donde no tengan que interactuar con ellos, ya que los asocian con la inducción a las drogas y la agresión física o verbal. En otros casos, 
la interacción con este sector implica el adquirir los conocimientos y técnicas de sobrevivencia por parte de los adultos, resultado de un proceso de inducción y pertenencia al grupo; además, de la protección ante otros riesgos y hostigamiento de otros actores, por parte del colectivo (la banda).

${ }^{8}$ El concepto de poblaciones callejeras, ha sido conceptualizado por Juan Martín Pérez y la organización El Caracol, A.C. y se define de la siguiente manera: "significa reconocer el carácter activo de las y los más pobres y excluidos de la estructura social de un país como México, es decir, grupos humanos que sobreviven, con sus propios recursos, en medio de las adversidades de la calle. Además, esta categoría social permite acercarse a una demografía diversa y cambiante. La particularidad de esta población, está en la construcción de su identidad en torno a la calle y la vulnerabilidad social en la que se encuentra el ejercicio de sus derechos. Esta categoría social está colaborando en la incorporación de nuevas miradas para repensar "las infancias", "la exclusión", "la discriminación", "la tutela”, "la demografía", "la cultura" y la "identidad", entre otros temas sociales" (CDHDF et. al., 2010:.2) 


\section{Consideraciones finales}

La niñez y adolescencia trabajadora en las calles configura sus interacciones con una amplia gama de actores gubernamentales, sociales, privados, familiares y sus pares. Cada interacción representa la posibilidad de establecer relaciones de cooperación y conflicto en el espacio público, que responden a los intereses y necesidades específicas del grupo social, delineadas por la lucha de la sobrevivencia cotidiana, en el acceso a ingresos diferenciados y los satisfactores inmediatos.

Como actores sociales en las calles, incorporan en sus prácticas sociales una serie de conocimientos, experiencias y habilidades para desplazarse y conocer la dinámica del entorno social. Con ello, identifican a la diversidad de actores en el espacio público y la producción de estrategias de resistencia específicas en sus interacciones. Toman decisiones y se organizan colectivamente para la elección y movilidad de las zonas de trabajo, en base a la reducción de los riesgos sociales y sortear los controles tutelares proteccionistas.
El reconocimiento como grupo social se configura en la identidad de su condición de marginación y exclusión social y por la posición que ocupan en la organización colectiva, familiar y asociativa, desde la óptica del micro espacio social, amplía los umbrales de su autonomía para la toma de decisiones respecto a su actividad económica, el acceso y manejo de sus ingresos en el espacio familiar y el auto-reconocimiento por su capacidad y habilidades de sobrevivencia en contextos hostiles y adversos.

Acercarnos a la vida de las niñas, niños y adolescentes que trabajan en las calles, implica algunas premisas: cuestionar y develar nuestra visión adultista sobre la infancia, lo cual permitiría construir nuevas formas de interacción no asimétricas con la niñez y adolescencia, reconociendo que, tanto adultos como niños, somos influidos por las mismas fuerzas del sistema.

Reconocer la diversidad de infancias amplía el horizonte, en la comprensión de prácticas complejas de diversos grupos sociales, en contextos culturales 
e históricos específicos. Más allá de elaboraciones de leyes, políticas y programas de protección a los derechos de la infancia-basadas en el supuesto adulto-,es necesario establecer un puente de diálogo que ponga énfasis en la resignificación de las prácticas y experiencias sobre la protección y participación que producen y requieren las niñas, niños y adolescentes que trabajan en las calles.

Apuntar al reconocimiento de la posición social de niñas, niños y adolescentes trabajadores en los ámbitos sociales, comunitarios, familiares y escolares a partir de acompañar y compartir su capacidad de agencia y protagonismo infantil.

Finalmente, agradezco el apoyo de las niñas, niños, adolescentes y jóvenes trabajadores en las calles que participaron en la investigación, quienes me brindaron su confianza y me permitieron conocer sus prácticas cotidianas en sus espacios familiares y sociales. 


\section{Referencias}

Aries, P. (1987). El niño y la vida familiar en el Antiguo Régimen. Madrid: Taurus

Bourdieu, Pierre (1990). Sociología y Cultura. México, Girjalbo.

Comisión de Derechos Humanos del DF (2010). Derechos de las poblaciones callejeras, México: CDHDF.

Comisión de Derechos Humanos del DF (2012). Limpieza social: respuesta falaz ante la crisis social, en revista Dfensor, $4(X)$.

Foucault, M. (1994). Un diálogo sobre el poder y otras conversaciones. Madrid: Alianza 2000.

Foucault, M. (2006). Seguridad, territorio, población. Buenos Aires: Fondo de Cultura Económica

Foucault, M. (2008). Historia de la Sexualidad: La Voluntad de Saber. Buenos Aires: Siglo XXI.

Gaitán, L. (2006). La nueva sociología de la infancia. Aportes de una mirada distinta. Revista. Política y Sociedad 1(43), pp.9-26.

Hardt, M. y Negri, T. (2000). Imperio. EUA: Harvard University Press.

Jodelet, D. (2008) El movimiento de retorno al sujeto y el enfoque de las representaciones sociales, en Cultura y Representaciones Sociales, 3 (5), pp.3263

Liebel, M. (2003). Infancia y Trabajo, Peru: IFEJANT.

Parsons, T. (1959). El sistema social. Madrid: Revista de Occidente.

Pilotti, F. (2001). Globalización y Convención sobre los Derechos del Niño: el contexto del texto. Chile: Naciones Unidas.

Wacquant, Loic (2006). Castigar a los parias urbanos, Antípoda (2), ISSN 19005407, pp.59-66. 


\section{Apéndice}

\section{Testimonios}

Niñas, niños y adolescentes participantes:

Niña 1, 8 años, vende rosas

Niña 2, 10 años, vende rosas y dulces.

Niña 3, 8 años, vende rosas y dulces.

Niña 4, 9 años, vende dulces.

Adolescente varón 1, 15 años, limpiaparabrisas.

Adolescente mujer 2, 12 años, vende rosas.

Adolescente mujer 3, 15 años, vende tamales

\section{Para citar este artículo:}

Narváez, (2019).

Las interacciones de la niñez

y adolescencia trabajadora en las

calles de Xalapa, Veracruz, México:

análisis de los mecanismos de

control y estrategias de resistencia.

Revista Educa UMCH, 13(1).

https://doi.org/10.35756/educaumch.v7i13.91 